\title{
LOW-GRADE FIBROMYXOID SARCOMA ARISING IN THE MEDIASTINUM
}

\author{
Iwao Takanami, MD, Ken Takeuchi, MD, and Masao Naruke, MD, Tokyo, Japan
}

In 1987 Evans $^{1}$ described 2 metastasizing soft tissue tumors with deceptively benign histologic appearance that he named low-grade fibromyxoid sarcoma (LGFM). Twentynine cases of LGFM have since been reported in the literature ${ }^{1-5}$ but no report has been published of the condition arising in the mediastinum. We describe a patient with a deceptively benign-appearing LGFM arising in the mediastinum, which recurred 9 years after original resection.

Clinical summary. In February 1990, a routine screening chest x-ray film of a subjectively healthy 35 -year-old man revealed a tumor in the anterior portion of the left mediastinum that had been gradually enlarging for 7 years. Through a median sternotomy, the mass, measuring $9.0 \times 5.5$ $\times 3.0 \mathrm{~cm}$ and well capsulated in the anterior mediastinum, was excised. No other intrathoracic and mediastinal tumors were noted during the operation. The mass was diagnosed as a "neurofibroma." The postoperative period was uneventful, and the subject remained well for 9 years. At that point, $x$-ray films of this symptom-free patient revealed a mass in almost the same part of the anterior mediastinum, estimated to be 6.0 $\times 5.5 \mathrm{~cm}$ in diameter (Fig 1). Computed tomography indicated a round, solid mass in the anterior part of the mediastinum adjacent to the sternum and left clavicle. Phlebogram showed complete blockage of the left innominate vein. At operation, the mediastinum was explored by cutting the clavicle and left first rib, and the tumor, measuring $6.0 \times 5.5 \times 5.0 \mathrm{~cm}$, involved the left innominate vein and the posterior part of the manubrium of the sternum. The left innominate vein was ligated, and the tumor was excised with the left half of the manubrium of the sternum and the head of the left clavicle and partial left first rib. Primary closure was performed without chest wall reconstruction. Histologically, the primary lesion was re-examined and compared with the local recurrent tumor, and the 2 specimens were found to be almost identical. Microscopically, the neoplasm was characterized by bland-appearing fibroblastic spindle cells in fibrous and myxoid areas (Fig 2). Slight nuclear pleomorphism and rare mitotic figures were observed. The arrangement of the cells was mostly a whorled pattern. These pathologic findings sug-

From the Department of Surgery, Teikyo School of Medicine, Tokyo, Japan.

Received for publication May 25, 1999; accepted for publication July 7, 1999

Address for reprints: Iwao Takanami, MD, Department of Surgery, Teikyo School of Medicine, 2-11 Kaga 2-Chome, Itabashi-Ku, Tokyo, 173 Japan.

J Thorac Cardiovasc Surg 1999;118:970-1

Copyright (C) 1999 by Mosby, Inc.

$0022-5223 / 99 \$ 8.00+0 \quad \mathbf{1 2 / 5 4 / 1 0 1 2 8 8}$

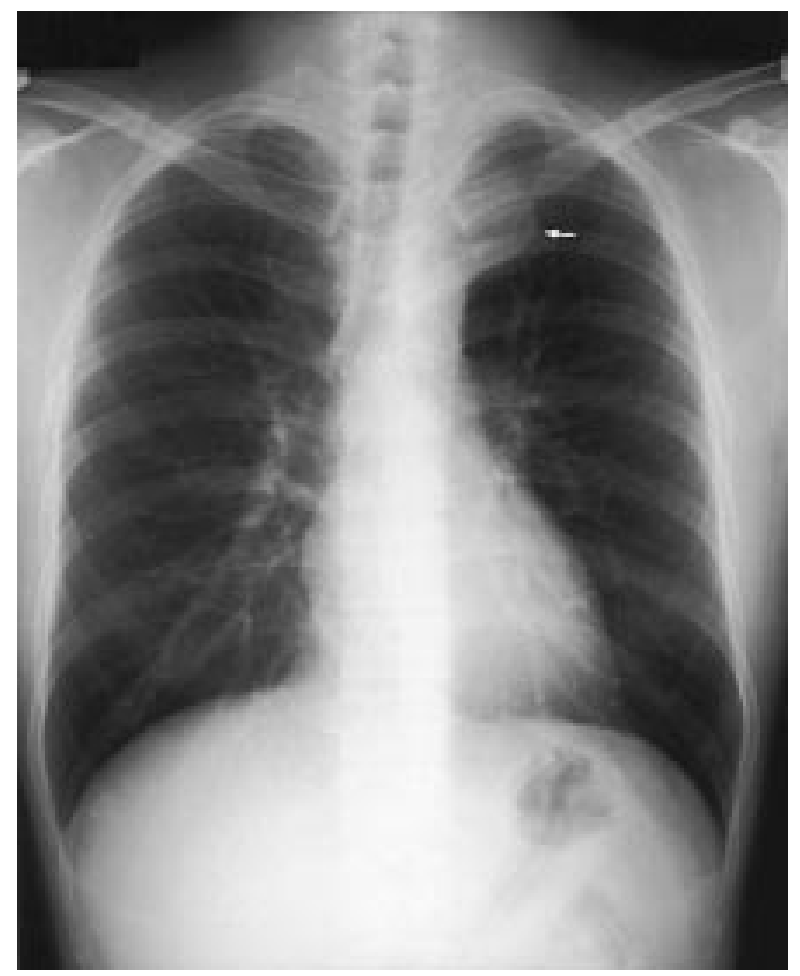

Fig 1. Chest x-ray film 9 years after resection of the anterior mediastinal tumor.

gest that the tumor was an LGFM. The patient was alive with no evidence of disease 2 months after excision.

Discussion. Benign soft tissue lesions may histologically mimic soft tissue sarcomas, and the reverse can also occur with sarcomas being mistaken for benign lesions. Primary lesions are diagnosed as neurofibromas by some pathologists after the initial operation and, although there may be multiple neurofibromas, there have been no reports of recurrent neurofibromas. Thus primary lesions have been histologically reexamined and compared with the local recurrent lesion. LGFM is a recently recognized, uncommon "benign appearing" tumor with a tendency to develop in the deep soft tissue of young adults. LGFM is reported to be often misdiagnosed as neurofibroma or desmoid fibromatosis. ${ }^{4}$ Grossly, the neoplasms have been reported to be large with well-defined margins. The original tumor and recurrent tumor in our case were respectively large and encapsulated with well-defined margins. Histologically, LGFMs have been characterized by bland spindle cells, showing a mainly whorled or focally linear arrangement, and set in alternating areas with a fibrous or myxoid stroma. ${ }^{5}$ The present tumor arising in the medi- 


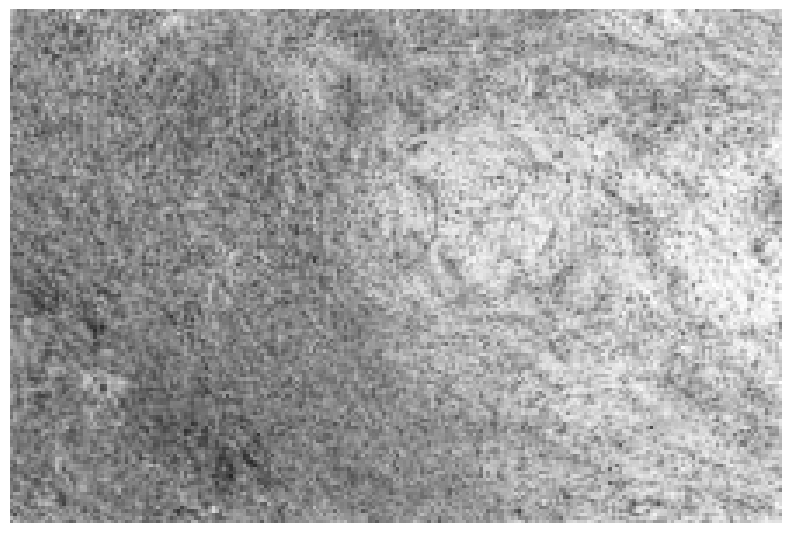

Fig 2. Histologic study of a low-grade fibromyxoid sarcoma, demonstrating contrasting fibrous and myxoid areas and a swirling, whorled growth pattern (recurrent tumor).

astinum fulfills Evans' criteria for LGFM. Soft tissue in the lower limbs and chest wall have been the most common primary sites of LGFM, ${ }^{4,5}$ and although there have been 2 cases of intra-abdominal tumors, ${ }^{4,5}$ there has been no report until now of LGFM arising in the mediastinum.

The course of the LGFM is unpredictable. The tumors usually follow a protracted clinical course with frequent recurrences. Among 29 reported cases, 16 showed local recurrence and 9 had lung metastases. The interval to local recurrences ranged from 2 years to 13 years (mean, 5 years), and the interval to lung metastasis ranged from zero to 45 years (mean, 5 years). The majority of subjects in the literature with adequate follow-up were still alive 10 years after primary excision of the lesion. Four of 12 patients in Evans' report ${ }^{4}$ died of their neoplasms, but in other authors' reports there were no deaths among 17 patients, although some of them had recurrences and/or metastasis. Survival may be prolonged in the presence of recurrences or metastases.

In conclusion, the possibility of LGFM must be taken into account in young adults who have a large mass characterized by fibrous and myxoid areas, even in the mediastinum. Close follow-up for a prolonged period is required.

\section{REFERENCES}

1. Evans HL. Low grade fibromyxoid sarcoma: a report of two metastasizing neoplasms having a deceptively benign appearance. Am J Clin Pathol 1987;88:615-9.

2. Fukunaga M, Ushigome S, Fukunaga N. Low-grade fibromyxoid sarcoma. Virchows Arch 1996;429:301-3.

3. Dvornik G, Barbareschi M, Gallotta P, Palma PD. Low grade fibromyxoid sarcoma. Histopathology 1997;30:274-6.

4. Evans HL. Low-grade fibromyxoid sarcoma: a report of 12 cases. Am J Surg Pathol 1993;17:595-600.

5. Goodlad JR, Mentzel T, Fletcher CDM. Low grade fibromyxoid sarcoma: clinicopathological analysis of eleven new cases in support of a distinct entity. Histopathology 1995;26:229-37. 